\title{
PEDF promotes the repair of bone marrow endothelial cell injury and accelerates hematopoietic reconstruction after bone marrow transplantation
}

Wen Ju' ${ }^{1,2,3 \dagger}$, Wenyi Lu ${ }^{1,2,3 \dagger}$, Lan Ding ${ }^{1,2,3 \dagger}$, Yurong Bao ${ }^{1,2,3}$, Fei Hong ${ }^{1,2,3}$, Yuting Chen ${ }^{1,2,3}$, Hui Gao ${ }^{1,2,3}$, Xiaoqi Xu ${ }^{1,2,3}$,

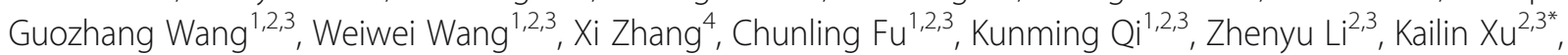
Jianlin Qiao ${ }^{1,2,3^{*}}$ and Lingyu Zeng ${ }^{1,2,3^{*}}$

\begin{abstract}
Background: Preconditioning before bone marrow transplantation such as irradiation causes vascular endothelial cells damage and promoting the repair of damaged endothelial cells is beneficial for hematopoietic reconstitution. Pigment epithelium-derived factor (PEDF) regulates vascular permeability. However, PEDF's role in the repair of damaged endothelial cells during preconditioning remains unclear. The purpose of our study is to investigate PEDF's effect on preconditioning-induced damage of endothelial cells and hematopoietic reconstitution.

Methods: Damaged endothelial cells induced by irradiation was co-cultured with hematopoietic stem cells (HSC) in the absence or presence of PEDF followed by analysis of HSC number, cell cycle, colony formation and differentiation. In addition, PEDF was injected into mice model of bone marrow transplantation followed by analysis of bone marrow injury, HSC number and peripheral hematopoietic reconstitution as well as the secretion of cytokines (SCF, TGF- $\beta$, IL-6 and TNF-a). Comparisons between two groups were performed by student t-test and multiple groups by one-way or two-way ANOVA.

Results: Damaged endothelial cells reduced HSC expansion and colony formation, induced HSC cell cycle arrest and apoptosis and promoted HSC differentiation as well as decreased PEDF expression. Addition of PEDF increased CD144 expression in damaged endothelial cells and inhibited the increase of endothelial permeability, which were abolished after addition of PEDF receptor inhibitor Atglistatin. Additionally, PEDF ameliorated the inhibitory effect of damaged endothelial cells on HSC expansion in vitro. Finally, PEDF accelerated hematopoietic reconstitution after bone marrow transplantation in mice and promoted the secretion of SCF, TGF- $\beta$ and IL-6.
\end{abstract}

Conclusions: PEDF inhibits the increased endothelial permeability induced by irradiation and reverse the inhibitory effect of injured endothelial cells on hematopoietic stem cells and promote hematopoietic reconstruction.

Keywords: PEDF, Endothelial cells, Hematopoietic stem cells, Hematopoietic reconstitution

\footnotetext{
* Correspondence: lihmd@163.com; jianlin.qiao@gmail.com;

zengly2000@163.com

${ }^{+}$Wen Ju, Wenyi Lu and Lan Ding contributed equally to this work.

${ }^{2}$ Key Laboratory of Bone Marrow Stem Cell, Jiangsu Province, Xuzhou, China

'Blood Diseases Institute, Xuzhou Medical University, Xuzhou, China

Full list of author information is available at the end of the article
}

C C The Author(s). 2020 Open Access This article is licensed under a Creative Commons Attribution 4.0 International License, which permits use, sharing, adaptation, distribution and reproduction in any medium or format, as long as you give appropriate credit to the original author(s) and the source, provide a link to the Creative Commons licence, and indicate if changes were made. The images or other third party material in this article are included in the article's Creative Commons licence, unless indicated otherwise in a credit line to the material. If material is not included in the article's Creative Commons licence and your intended use is not permitted by statutory regulation or exceeds the permitted use, you will need to obtain permission directly from the copyright holder. To view a copy of this licence, visit http://creativecommons.org/licenses/by/4.0/ The Creative Commons Public Domain Dedication waiver (http://creativecommons.org/publicdomain/zero/1.0/) applies to the data made available in this article, unless otherwise stated in a credit line to the data. 


\section{Background}

Hematopoietic stem cell transplantation (HSCT) is widely used for treating hematological malignancies [13]. However, long-term hypoglycemia after transplantation, that is, poor graft function (PGF), seriously affects patient survival and quality of life [4, 5]. Studies have shown that PGF is closely related to the hematopoietic microenvironment [6]. Therefore, in-depth exploration of microenvironmental factors affecting HSC homing and implantation, and accelerating hematopoietic reconstruction and hematopoietic function recovery after HSCT are potential research directions in the field of HSCT transplantation.

Hematopoietic microenvironment is an internal environment that regulates and supports the growth and development of hematopoietic cells. It is mainly composed of stromal cells and extracellular matrix [7, 8]. Among them, endothelial cells are an important part of the hematopoietic microenvironment and involved in hematopoietic reconstruction [9-11]. Our previous study found that infusion of endothelial progenitor cell (EPC) can reduce the incidence and severity of graftversus-host disease (GVHD) and promote hematopoietic reconstruction after HSCT [12]. EPC can differentiate into endothelial cells and promote the repair of injured vascular niche, indicating its important role in hematopoietic reconstruction [13]. Under normal conditions, mature vascular endothelium is in a stable quiescent state, but under pathological conditions, the vascular endothelium is detached, leading to morphology changes, increased vascular permeability and vascular fibrosis [14-16]. We previously found that preconditioning regimens prior to HSCT could cause damage to vascular endothelial structure and function and changes in endothelial permeability [17-19]. However, the current underlying mechanisms of endothelial injury and strategies to promote endothelial repair during preconditioning treatment are still lacking [14].

Pigment epithelium-derived factor (PEDF) is a $50-\mathrm{kDa}$ non-inhibitory factor in the serine protease inhibitor gene family and secreted by vascular endothelial cells, pericytes and retinal pigment epithelial cells [20]. Several studies have shown that PEDF is closely related to the function of vascular endothelial cells and exerts multiple effects such as anti-inflammation, antioxidant, antitumor, anti-angiogenesis, and inhibition of vascular permeability [21-25]. PEDF can inhibit vascular endothelial growth factor (VEGF) and stress-induced increase in vascular permeability in vitro and in vivo [21, 26-28]. PEDF regulates vascular permeability by preventing the dissociation of $\mathrm{AJ}$ and $\mathrm{TJ}$ proteins and regulating $\mathrm{AJ}$ protein phosphorylation via $\gamma$-secretase pathway [29]. PEDF has also been reported to prevent increased vascular permeability caused by hypoxia stress [21] and protect ox-
LDL-induced endothelial cell damage by inhibiting the Wnt signaling pathway [30]. However, it is unknown whether PEDF could repair the damaged endothelium and promote hematopoietic reconstruction during preconditioning process. Our study aims to investigate the effect of PEDF on injured endothelial repair and hematopoietic reconstruction during preconditioning to provide new ideas for reducing PGF and accelerating hematopoietic reconstruction.

\section{Methods \\ Cell culture}

bEnd-3 (ATCC ${ }^{\otimes}$ CRL-2299 ${ }^{\mathrm{TM}}$ ) were used between the fourth and tenth passage and cultured in Dulbecco's Modified Eagle Medium (DMEM, Gibco, catalog number: C11995500BT) supplemented with 10\% fetal bovine serum (FBS, Gibco, catalog number: 10099-141).

\section{Irradiation injury cell model and grouping}

Endothelial cells (EC) $\left(1 \times 10^{5}\right.$ per well in 6-well plate) received irradiation using GSR C1 137 caesium gamma irradiator (Gamma-Service Medical, Bautzner, Germany) at a dose of $15 \mathrm{~Gy}$ with a dose rate of $1.88 \mathrm{~Gy} / \mathrm{min}$ and cultured at $37{ }^{\circ} \mathrm{C}$ incubator for $72 \mathrm{~h}$.

PEDF + 15 Gy EC group: $6 \mathrm{~h}$ before irradiation, endothelial medium containing recombinant mouse PEDF protein(100 ng / ml, sangon biotech, China) was added into cells followed by receiving irradiation.

PEDF + Atglistatin group: ATGL inhibitor Atglistatin (10 $\mu \mathrm{M}$, MedChemExpress, CAS No.: 1469924-27-3) was added $1 \mathrm{~h}$ before PEDF administration and $6 \mathrm{~h}$ before irradiation, endothelial medium containing PEDF $(100 \mathrm{ng} / \mathrm{ml})$ was added followed by receiving irradiation.

\section{Primary HSC culture}

Mouse bone marrow cells (BMC) were collected using Lin-sorting (EasySep ${ }^{\mathrm{Tm}}$ Mouse Hematopoietic Progenitor Cell Isolation Kit, STEM CELL, Catalog \# 19856) and CD117 sorting (CD117 MicroBeads, MACS, Cat \# 130091-224). Endothelial cells were inoculated in 24-well plates at a density of $2 \times 10^{4} / \mathrm{ml}$ per well. After adherence for $24 \mathrm{~h}$, endothelial cells were damaged by Busulfan $(0.06 \mu \mathrm{g} / \mu \mathrm{l})$. The next day, isolated bone marrow cells (BMC) was inoculated according to the optimal culture ratio (b3: $\mathrm{HSC}=1: 6$ ) and cultured. Primary HSC medium consisted of Stem $\operatorname{Span}^{\text {тм }}$ SFEM (STEM CELL, catalogue number: 09650), SCF (20 ng / ml) (Peprotech, catalog number: 250-03), TPO (20 ng / ml) (Peprotech, catalogue number 315-14), Pen Strep (Gibco, catalogue number: 15140-122). PEDF (100 ng / ml) was added into cocultured medium. 


\section{Experimental animals}

SPF male inbred C57BL/6 mice aged 6-8 weeks were purchased from Beijing Weitong Lihua Experimental Technology Co., Ltd. and raised at Experimental Animal Center. This study was approved by the Animal Ethics Committee of Xuzhou Medical University.

\section{qRT-PCR}

RNA was isolated from endothelial cells followed by cDNA synthesis for measuring the expressions of occludin and Zona occludens proteins 1 (ZO-1) by qRT-PCR. The primer sequences were as follows:

Occludin-F-5' -TTGAAAGTCCACCTCCTTACAGA-3'; Occludin-R-5' -CCGGATAAAAAGAGTACGCTGG-3' . ZO-1-F-5' -GCTTTAGCGAACAGAAGGAGC-3'; ZO-1R-5' -TTCATTTTTCCGAGACTTCACCA-3'.

PEDF-F-5' -GCCCTGGTGCTACTCCTCT-3'; PEDF-R-5' -CGGATCTCAGGCGGTACAG-3'. $\beta$-actin-F-5' -ATGTGGATCAGCAAGCAGGA-3'; $\beta$ actin-R-5' -AAGGGTGTAAAACGCAGCTCA-3' .

\section{PEDF-siRNA transfection}

PEDF shRNA lentiviral plasmids were constructed using GV248 plasmid (hU6-MCS-Ubiquitin-EGFP-IRES-puromycin, Genechem) the sequences of Easy-siRNA are designed as follows, the oligo synthesis information of PEDF-shRNA (88706-1, sh6): 5'-CCGGGCCCAGAT GAAAGGGAAGATTCTCGAGAATCTTCCСTTTCAT CTGGGCTTTTTG-3'; 5' -AATTCAAAAAGCCCAG ATGAAAGGGAAGATTCTCGAGAATCTTCCCTTTC ATCTGGGC-3'.

Sh6 targeted sequence was GCCCAGATGAAAGG GAAGATT.

PEDF-shRNA (88707-1, sh7): 5'-CCGGGGCCAT CTTACGATACGGCTTCTCGAGAAGCCGTATCG TAAGATGGCCTTTTTG-3'; 5' -AATTCAAAAAGGC CATCTTACGATACGGCTTCTCGAGAAGCCGTAT CGTAAGATGGCC-3'.

Sh7 targeted sequence was GGCCATCTTACGAT ACGGCTT.

Control (shctrl) sequence was TTCTCCGAACGTGT CACGT. Lentivirus were prepared followed the protocol of Genechem. Endothelial cells were cultured in antibiotic-free growth medium to reach about $50 \%$ confluence and infected following the company's experimental procedures of Genechem. Positive cells were selected using puromycine.

\section{Western blot}

The expressions of occludin were detected in endothelial cells by western blot. Total protein was isolated, separated on $10 \%$ SDS-PAGE, and transferred to PVDF membrane. After blocking with $5 \%$ BSA for $2 \mathrm{~h}$, the membrane was incubated with the primary antibodies against occludin (1: 1000, servicebio, catalogue number: GB11149-2), GAPDH (1: 1000, servicebio, catalogue number: GB11002) at $4{ }^{\circ} \mathrm{C}$ overnight followed by washing with TBST and incubation with HRP-labeled goat anti-rabbit secondary antibody (1: 5000, servicebio, catalogue number: GB23303) for $1 \mathrm{~h}$. The membrane was developed after addition of ECL reagent.

\section{HE staining}

Mice were sacrificed and femurs were isolated. After fixation decalcification, dehydration and paraffin embedding, femurs were sliced into $4 \mu \mathrm{m}$ thickness and stained with HE Solutions. The pathologic changes of bone marrow were estimated by the optical microscope (Nikon).

\section{Immunofluorescence staining}

Cells or frozen sections were fixed in $4 \%$ paraformaldehyde for $1 \mathrm{~h}$, blocked with 5\% BSA containing 0.3\% Triton $\mathrm{X}-100$ for $1 \mathrm{~h}$, and incubated with primary antibody against CD144 (1: 500, Abcam, catalogue number: ab205336) at $4{ }^{\circ} \mathrm{C}$ overnight followed by washing 3 times with $\mathrm{PBS}$ and incubation with anti-rabbit $\operatorname{IgG}(\mathrm{H}+\mathrm{L}), \mathrm{F}$ (ab ') 2 Fragment (Alexa Fluor 488 Conjugate) (1: 500, CST, catalogue number: $4412 \mathrm{~S})$ for $2 \mathrm{~h}$. After washing in PBS three times, DIPA $(1 \mathrm{mg} / \mathrm{ml})$ was added for $10 \mathrm{~min}$ incubation followed by washing twice with PBS, mounting on an anti-tissue fluorescence quencher and observation under a microscope.

Mice femurs were isolated and keeped in 4\% PFA for $2 \mathrm{~h}$ at $4{ }^{\circ} \mathrm{C}$. After decalcification and dehydration, the femurs were embedded with $8 \%$ gelatin $20 \%$ sucrose $2 \%$ PVP and kept at $-80^{\circ} \mathrm{C}$. Femurs were sliced into 20$30 \mu \mathrm{m}$ thickness. Non-specific binding were blocked with $4 \% \mathrm{BSA}$ in PBS at room temperature for $1 \mathrm{~h}$. The desired primary antibody diluted in PBS with $1 \%$ BSA were covered the sections at $4{ }^{\circ} \mathrm{C}$ overnight. For bone marrow endothelial staining, we used Mouse Endomucin Antibody (RD, AF4666). Rat anti-CD150 (Biolegends, 115, 902), Rat anti-CD48 (Biolegends, 103,432), Rat antilineage-biotin (Biolegends), DyLight ${ }^{\oplus} 550$ (ThermoFisher, SA5-10027) and Streptavidin-Brilliant Violet 421 ${ }^{\mathrm{TM}}$ (Biolegends, 405,225) were used for HSC staining.

\section{Cell colony formation}

Prepare a cell suspension that is $10 \times$ the final plating concentration $\left(2 \times 10^{5}\right.$ cells $/ \mathrm{mL}$ for $2 \times 10^{4}$ cells / dish $)$ by diluting with Iscove's MDM (STEM CELL, catalogue number:07700) $+2 \%$ FBS. Add $300 \mu \mathrm{L}$ of cells to $3.0 \mathrm{~mL}$ of MethoCult ${ }^{\mathrm{Tm}}$ (STEM CELL, catalogue number: 03444) for duplicate dishes. The contents were mixed thoroughly. Let stand for at least $5 \mathrm{~min}$ to allow bubbles to rise to the top. Dispense MethoCult ${ }^{\mathrm{TM}}$ mixture into 35 $\mathrm{mm}$ dishes for methylcellulose-based cultures using a blunt-end needle attached to a syringe. Place the culture 
dishes in a large outer dish containing uncovered 100 $\mathrm{mm}$ dishes filled with sterile water to maintain proper humidity of MethoCult ${ }^{\mathrm{tw}}$ medium. Incubate cells for 10 days in a humidified incubator at $37^{\circ} \mathrm{C}$ and $5 \% \mathrm{CO}_{2}$.

\section{Cell cycle determination}

$2 \mu \mathrm{l}$ each of the corresponding Lin strains markers (Ter119, CD45R / B220, CD11b, CD8a, CD4, Gr-1), Ckit and Sca-1 was added to the cell suspension and incubated at $4{ }^{\circ} \mathrm{C}$ for $25 \mathrm{~min}$ followed by washing and addition of FIX \& PERM $^{\bullet}$ Sample Kit A (Nordic-MUbio, catalogue number: GAS-002 M) for $15 \mathrm{~min}$ incubation and subsequent addition of $100 \mu \mathrm{l}$ of FIX \& PERM $^{\circ}$ Sample Kit B (Nordic-MUbio, catalogue number: GAS-002 $\mathrm{M})$ and $30 \mu \mathrm{l}$ DIPA for overnight incubation at $4{ }^{\circ} \mathrm{C}$. Then, cell cycle was analyzed.

\section{Flow Cytometry}

Bone marrow cells were flushed from the femurs and tibias of mice with PBS containing 2\% FBS and lysed with lysing buffer (catalogue number: R1010, Solarbio, China) for red blood cell deletion. Bone marrow cells were suspended in PBS and stained by antibodies for 30 min. For Detection lineage-specific markers: Erythroid: Ter119 (FITC, Biolegend, catalogue number: 116206) and CD71 (PE, eBioscience, catalogue number:12-071182), Granular line: CD11b (FITC, BD, catalogue number: 557396) and Gr-1 (PE, BD, catalogue number: 553126), Megakaryocyte: CD41 (FITC, BD, catalogue number: 553848) and C-kit (PE, BD, catalogue number:553355), LSK: lineage cocktail (FITC), sca-1 (APC, Biolegend, catalogue number:108112) and C-kit (PE, BD, catalogue number:553355). Cell apoptosis analysis was performed using Annexin V staining kit according to the instructions of the manufacturer (BD).

\section{Cell permeability assessment}

Cells were seeded in the Transwell chamber $(200 \mu \mathrm{l})$ at a density of $2 \times 10^{4} / \mathrm{ml}$. After $72 \mathrm{~h}$, the upper and lower cells were washed with PBS, $200 \mu$ of FITc-Dextran (1 $\mathrm{mg} / \mathrm{ml}$ ) was added to the upper chamber and $200 \mu \mathrm{l}$ PBS was added to the lower chamber for $3 \mathrm{~h}$ incubation. $100 \mu \mathrm{l}$ of liquid was collected from the lower chamber for measuring the optical density $(\mathrm{OD})$ value. $\mathrm{Pa}=[\mathrm{A}] /$ $\mathrm{t} \times(1 / \mathrm{A}) \times(\mathrm{v} /[\mathrm{L}])$, where [A] is the FITC-Dextran concentration in the top chamber (expressed as fluorescence intensity), $\mathrm{t}$ is time (sec), $\mathrm{A}$ is the area of the filter membrane $\left(\mathrm{cm}^{2}\right)$, and $\mathrm{v}$ is the amount of liquid in the bottom chamber $(\mathrm{ml})$, [L] is FITC-Dextran concentration in the bottom chamber (expressed as fluorescence intensity). The results were expressed as the percentage change of $\mathrm{Pa}, \mathrm{Pa} \%=($ Experimental group $\mathrm{Pa}) /($ Control group $\mathrm{Pa}) \times 100 \%$.

\section{BMT model}

BMT mice were divided into PEDF + BMT group and BMT group and samples were collected at day 7, 14, 21, and 28 after bone marrow cell transplantation. Both groups of mice received an irradiation of $8.5 \mathrm{~Gy}$. PEDF group received intraperitoneal injection of PEDF recombinant protein at $0.5 \mathrm{mg} / \mathrm{kg} 12 \mathrm{~h}$ before irradiation and every 2 days afterwards. The BMC group received intraperitoneal injection of the same amount of PBS. The next day after receiving radiation, bone marrow cells of syngeneic mice were taken for HSC transplantation through injection of $5 \times 10^{6}$ bone marrow cells via tail vein.

\section{ELISA}

ELISA kits were used to detect the levels of SCF, TGF- $\beta$, IL- 6 and TNF- $\alpha$ in the cell culture supernatant and bone marrow supernatant.

\section{Statistical analysis}

All data were analyzed by GraphPad Prism 8.0 software and expressed as mean \pm standard deviation (SD). Comparisons between two groups were performed by $\mathrm{t}$-test and analysis of variance was used for comparison between multiple groups. $P<0.05$ indicates a significant difference.

\section{Results}

Injured endothelial cells affect HSC expansion

A large number of studies have demonstrated that bone marrow microvascular endothelium is severely damaged after HSCT, affecting hematopoietic reconstruction. However, the effect of damaged endothelial microenvironment on HSC is unknown. To investigate this, our study first established an in vitro co-culture model of Busulfan injured endothelial cells and HSC. It is known that vascular endothelial cells, as important hematopoietic stromal cells, can promote HSC proliferation which was confirmed by our study (Fig. 1a). Meanwhile, we found that co-culture of damaged endothelial cells and HSC inhibited HSC expansion (Fig. 1a), leading to a decrease in the proportion and total number of HSC (Fig. 1b). In addition, the ratio of G0 / G1 phase of HSC in co-culture group was increased, leading to reduced HSC proliferation (Fig. 1c) and increased apoptosis (Fig. 1d). Further analysis showed that the colony-forming ability in co-culture group was reduced (Fig. 1e). Consistently, the percentage of red blood cells, granulocytes, and megakaryocytes in the co-cultured group was increased significantly (Fig. 1f). The above results suggest that damaged endothelial cells inhibit HSC expansion in vitro. 


\section{A}
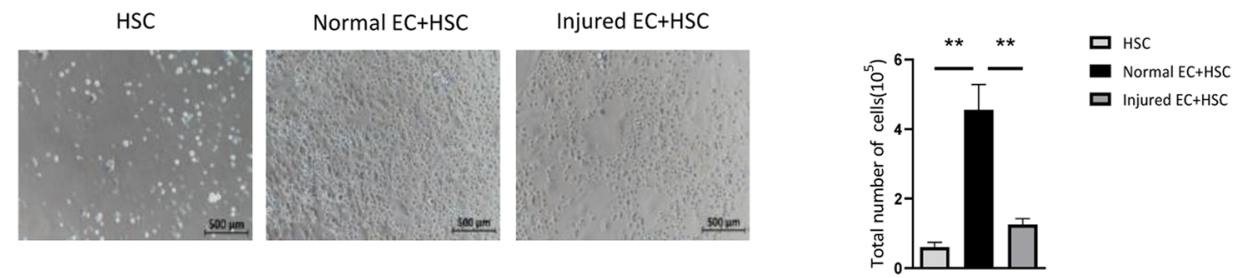

B In lin-
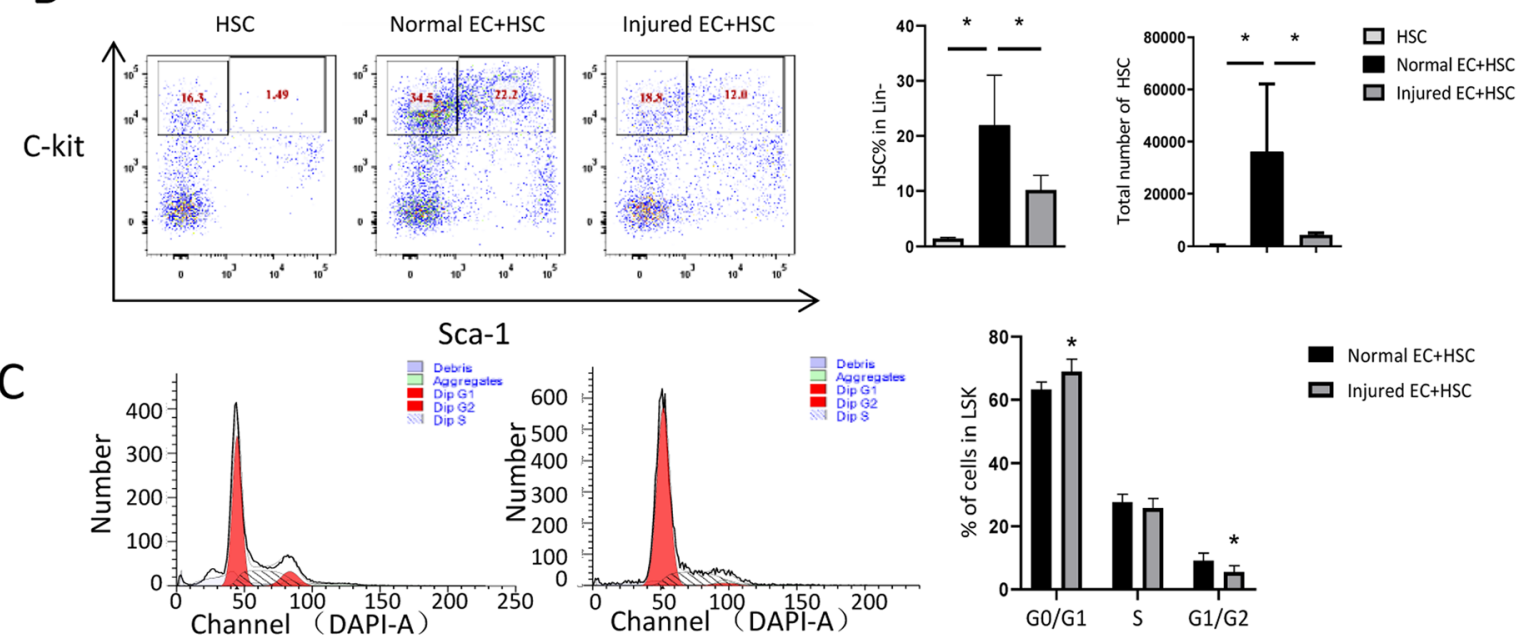

D

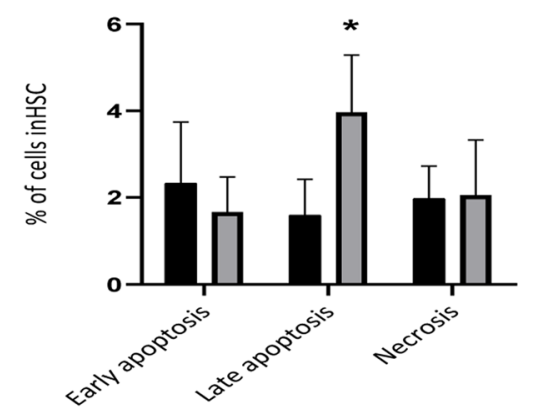

$\mathrm{E}$

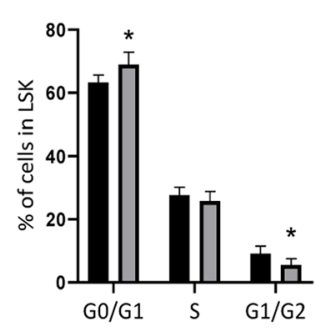

- Normal EC+HSC

口 Injured EC+HSC

Noraml EC+HSC

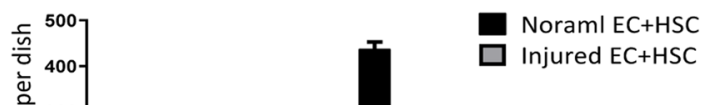

$\mathrm{F}$ Erythrocytosis Guanulocytosis Thrombocytosis

Normal EC+HSC
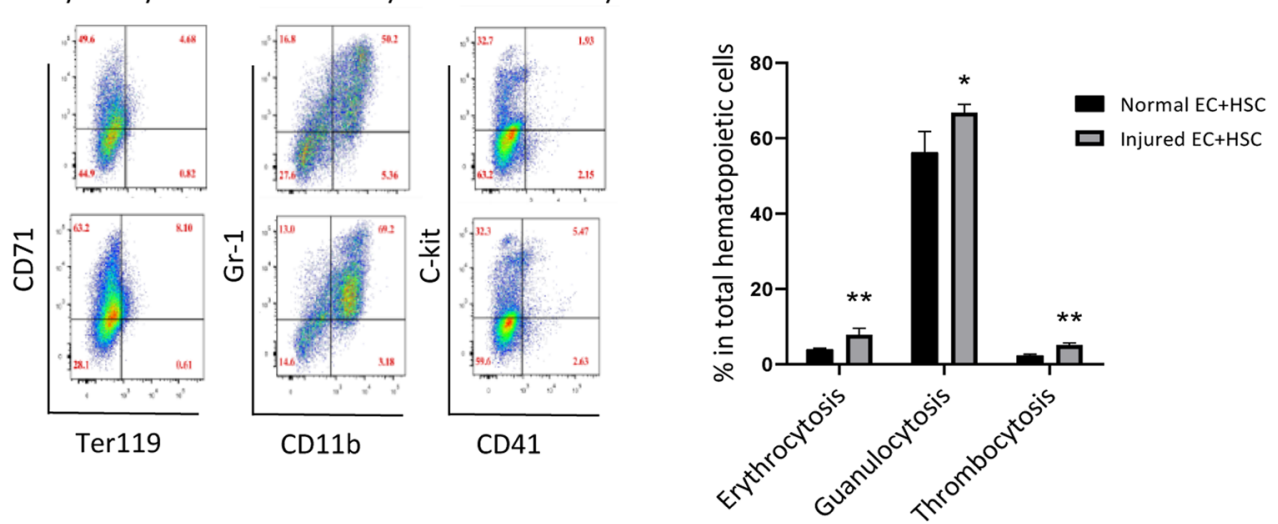

Fig. 1 (See legend on next page.) 
(See figure on previous page.)

Fig. 1 In vitro analysis of the effect of injured EC on HSC expansion, cell cycle, apoptosis and differentiation in a co-culture system. a Representative Microscopic observation of HSC expansion after cocultured with normal or damaged endothelial cells for 7 days; b Flow cytometry analysis of the proportion and number of HSCs after co-culture with normal endothelial cells or damaged endothelial cells. c HSC cell cycle analysis by flow cytometry; $\mathbf{d}$ HSC apoptosis analysis; e Colony-forming capacity of HSCs after co-culture; f Flow cytometry analysis of the proportion of erythrocytes, granulocytosis and thrombocytosis $\left(n=3-4,{ }^{*} p<0.05,{ }^{* *} p<0.01,{ }^{* * *} p<0.0001\right)$

\section{PEDF protects preconditioning-induced injury of endothelial cells in vitro}

We found that the level of pigment epithelium-derived factor (PEDF) was decreased significantly after endothelial cell injury byy-irradiation (Fig. 2a) without changes of vascular endothelial growth factor (VEGF) and platelet derived growth factor PDGF (data not shown). As PEDF is an endogenous functional protein with cytoprotective effects and closely related to endothelial cell permeability. Therefore, we tested whether PEDF has a protective effect on the damaged endothelial cells and found that CD144 expression was significantly reduced after irradiation and increased after addition of PEDF (Fig. 2b). Consistently, immunofluorescence assay also showed the increased expression of CD144 in endothelial cells after treatment with PEDF (Fig. 2c). In addition, further analysis confirmed that PEDF can inhibit the increase of endothelial permeability which is caused by irradiation and elevate the expression of occludin and $\mathrm{ZO}-1$, which are endothelial permeability related indicators (Fig. 2d). We also used PEDF shRNA to knock down PEDF expression to check PEDF's effect on permeability in the irradiation cell model and found that PEDF knock-down could inhibit the occludin mRNA and protein expression (Fig. 2e).

\section{PEDF improves the permeability of endothelial cells after} irradiation through ATGL receptors

To determine whether PEDF receptor Adipose triglyceride lipase (ATGL) is involved in PEDF's effect on the permeability of injured endothelial cells, we used the ATGL inhibitor Atglistatin and found that the increase of CD144 expression induced by PEDF was suppressed after addition of Atglistatin (Fig. 3a). In addition, Atglistatin can abolish the protective effect of PEDF on the permeability of injured endothelial cells (Fig. 3b). Moreover, the increase of occludin expression induced by PEDF was also inhibited (Fig. 3c). This data show that ATGL receptors are involved in the regulation of PEDF's effect on injured endothelial cell permeability.

\section{PEDF ameliorates the inhibitory effect of damaged endothelial cells on HSC expansion}

In order to determine whether PEDF can reverse the inhibitory effect of injured endothelial cells on HSC, PEDF was added to the co-culture system and our results showed that PEDF can significantly improve the reduced
HSC expansion ability induced by Busulfan damaged endothelial cells (Fig. 4a). Meanwhile, PEDF can increase the reduction of $\mathrm{HSC} \%$ and restore the number of HSCs in the co-culture system (Fig. 4b). Additionally, addition of PEDF can significantly inhibit the differentiation of HSC induced by damaged endothelial cells (Fig. 4c). The above results suggest that PEDF can ameliorate the inhibitory effect of injured endothelial cells on HSC and promote HSC expansion in vitro.

PEDF accelerates hematopoietic reconstitution after BMT To determine whether PEDF can promote hematopoietic reconstruction after $\mathrm{BMT}$, mouse PEDF recombinant protein was injected before and after BMT (Fig. 5a) and specimens were collected on day 7, 14, 21 and 28 after transplantation. Bone marrow pathology analysis on day 7, 14,21,28 suggested that hematopoietic recovery was gradually recovered after transplantation, while PEDF could reduce bone marrow pathological and endothelial damage (Fig. 5b) and accelerate hematopoietic reconstruction as demonstrated by improve recovery of the number of white blood cells and platelets (Fig. 5d-f). Bone marrow cell count results also showed that the number of bone marrow cells in the PEDF + BMT group was significantly higher than that in the BMT group at 14 days, 21 days, and 28 days after transplantation (Fig. 5c). Moreover, PEDF increased the proportion of erythrocytes and megakaryocytes and decreased myeloid cells (Fig. 5g-i). Furthermore, the proportion of hematopoietic stem progenitor cells (HSPC) was also significantly higher after PEDF addition (Fig. 5j and k). The immunostaining analysis of Lin-CD48-CD150+ HSC and bone marrow further suggest that PEDF promotes hematopoietic stem cell reconstruction (Fig. 5l) and the repair of bone marrow endothelial injury (Fig. 5b) after BMT.

\section{PEDF promotes the secretion of SCF, TGF- $\beta$ and IL- 6}

We next tested the levels of hematopoietic related factors SCF, TGF- $\beta$, IL- 6 and TNF- $\alpha$ and found that the levels of SCF, TGF- $\beta$ and IL- 6 were decreased and TNF$\alpha$ level was increased after endothelial cells were damaged and their levels were reversed after addition of PEDF (Fig. 6a) in the co-culture system. In vivo experiments further confirmed that PEDF can promote the secretion of SCF, TGF- $\beta$, and IL- 6 in the bone marrow supernatant of BMT mice and inhibit TNF- $\alpha$ secretion 


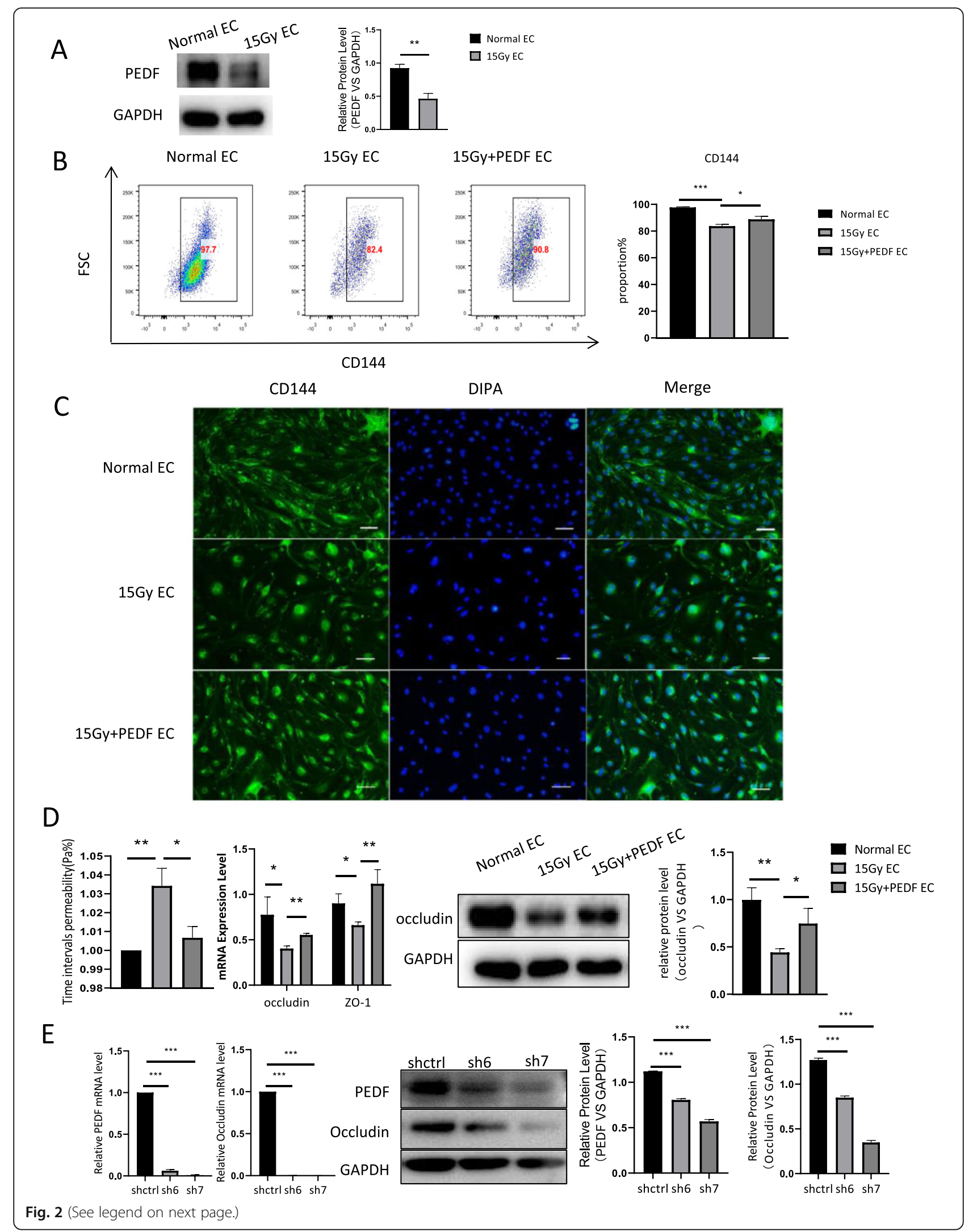


(See figure on previous page.)

Fig. 2 PEDF improves endothelial permeability. a Detection of PEDF level in irradiation injured endothelial cells by western blot. b Flow cytometry detected endothelial cell surface marker CD144 expression after irradiation. c Cell immunofluorescence ( $\times 100)$ analysis of CD144 expression. $\mathbf{d}$ Transwell assay analysis of endothelial cell permeability and the expression of occludin and ZO-1. e PEDF shRNA (sh6 and sh7) were used to knock down PEDF and check the occludin expression in injured endothelial cells after irradiation $\left(n=3,{ }^{*} p<0.05,{ }^{* *} p<0.01\right.$,

*** $p<0.001$ )

early after transplantation (Fig. 6b-e). The above results suggest that PEDF promotes hematopoietic reconstitution possibly through regulating these cytokines.

\section{Discussion}

Bone marrow microvascular endothelial cells are an important part of the bone marrow microenvironment and play an important role in maintaining bone marrow homeostasis and promoting hematopoietic reconstruction [12, 31, 32]. A large number of data have proved that radiotherapy and chemotherapy can cause severe damage to bone marrow microvascular endothelium [14, 33]. However, there are few reports on the underlying mechanism of endothelial injury and promoting bone marrow endothelial injury repair strategies after radiotherapy and chemotherapy. Our study profoundly elucidates the mechanism of PEDF in repairing damaged endothelial cells and promoting hematopoietic reconstruction.

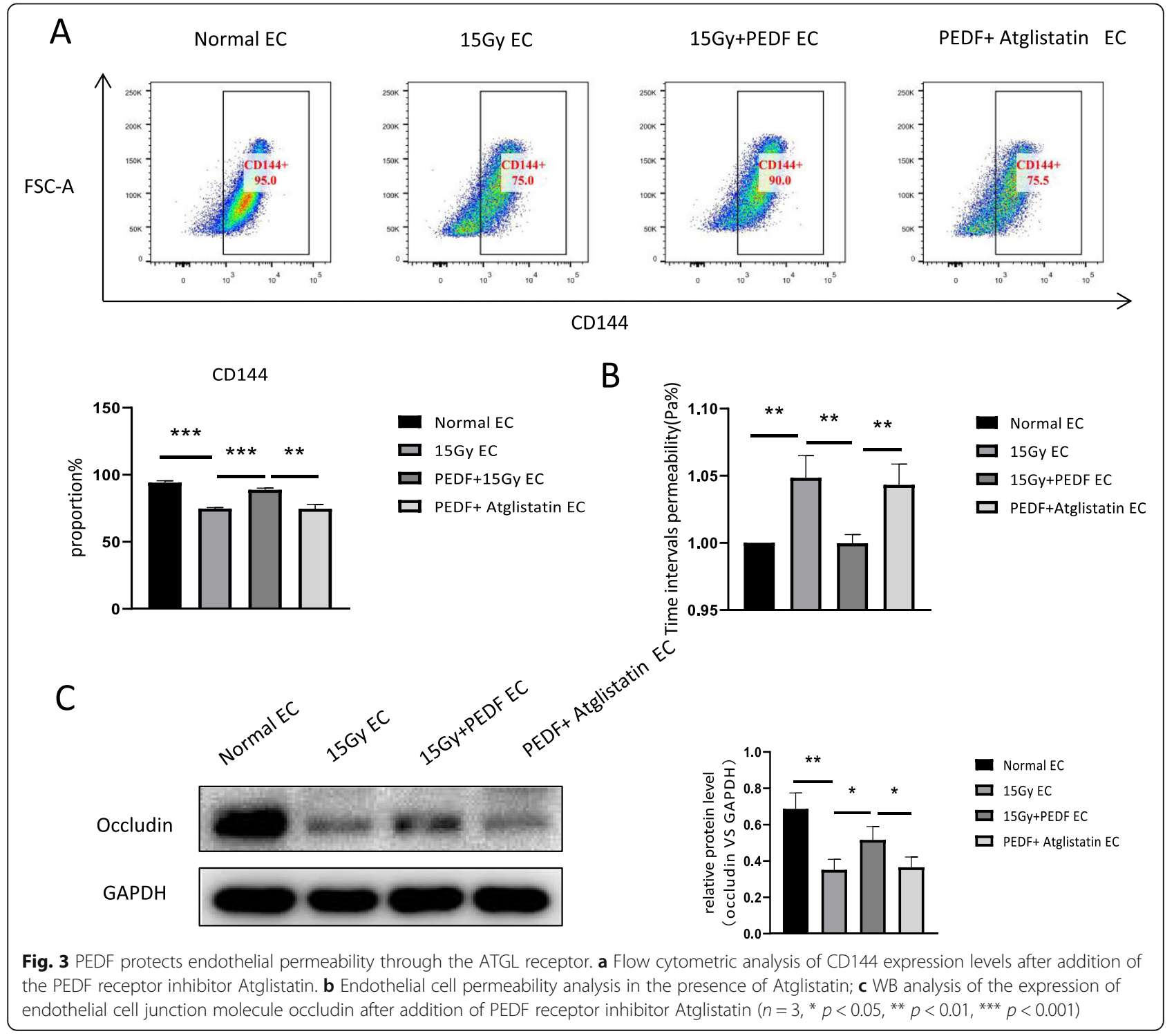




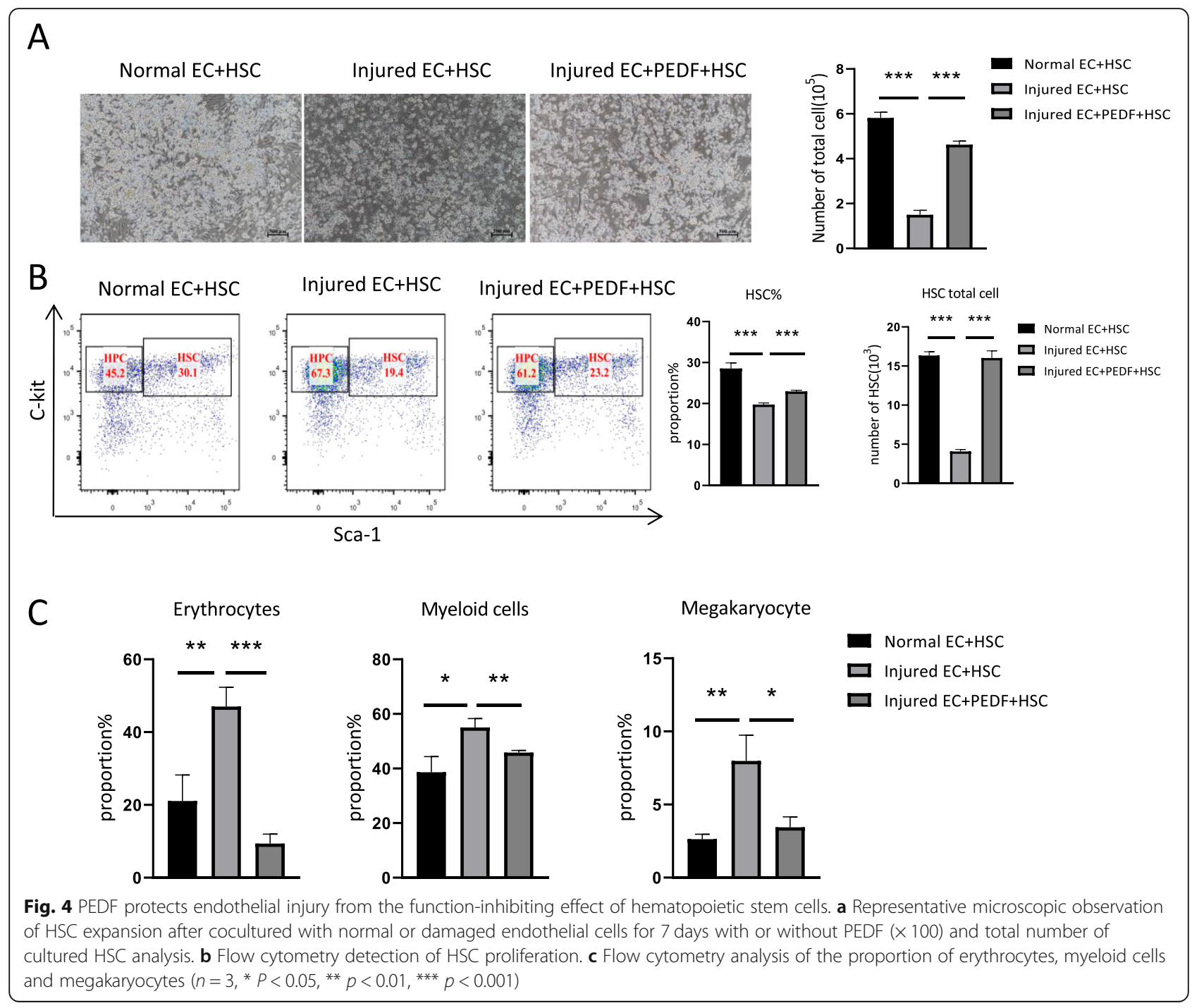

It is known that endothelial cells are important hematopoiesis-promoting stromal cells and promote the in vitro expansion of hematopoietic stem cells [10]. However, it is unknown whether injured endothelial cells can directly inhibit hematopoietic stem cells. Therefore, this study first established an in vitro microvascular endothelial injury model to study the effect of injured endothelial cells on the biological function of hematopoietic stem cells. We found that damaged endothelial cells can inhibit the expansion of hematopoietic stem cells in vitro, induce cell cycle arrest, apoptosis, and promote the differentiation of hematopoietic stem cells, suggesting that it has a direct inhibitory effect on hematopoietic stem cells. However, how to repair the damaged endothelial cells and ameliorate the effect of damaged endothelium on hematopoietic stem cells is unknown. Therefore, there is an urgent need to explore or discover drugs that can protect the endothelial cells and promote hematopoietic reconstruction.
Pigment epithelium-derived factor (PEDF) is a multifunctional protein secreted by a variety of cells. It has anti-inflammatory, anti-oxidant, anti-fibrotic, antivascular permeability enhancement, and cytoprotective effects without toxicities on hematopoietic stem cells [34]. It is a natural treatment strategy for endothelial injury with great potential and promising clinical application. In order to investigate whether PEDF can reverse the inhibitory effect of injured endothelial cells on the biological function of hematopoietic stem cells, we added PEDF to the co-culture system and found that PEDF can reverse the inhibitory effect of injured endothelial cells on hematopoietic stem cells and promote the expansion of hematopoietic stem cells in vitro, suggesting that PEDF has a protective effect on injured endothelial cells and can reverse the inhibitory effect of injured endothelial cells on hematopoietic stem cells.

Next, we found that PEDF injection can promote hematopoietic reconstruction after BMT in mice. Studies 


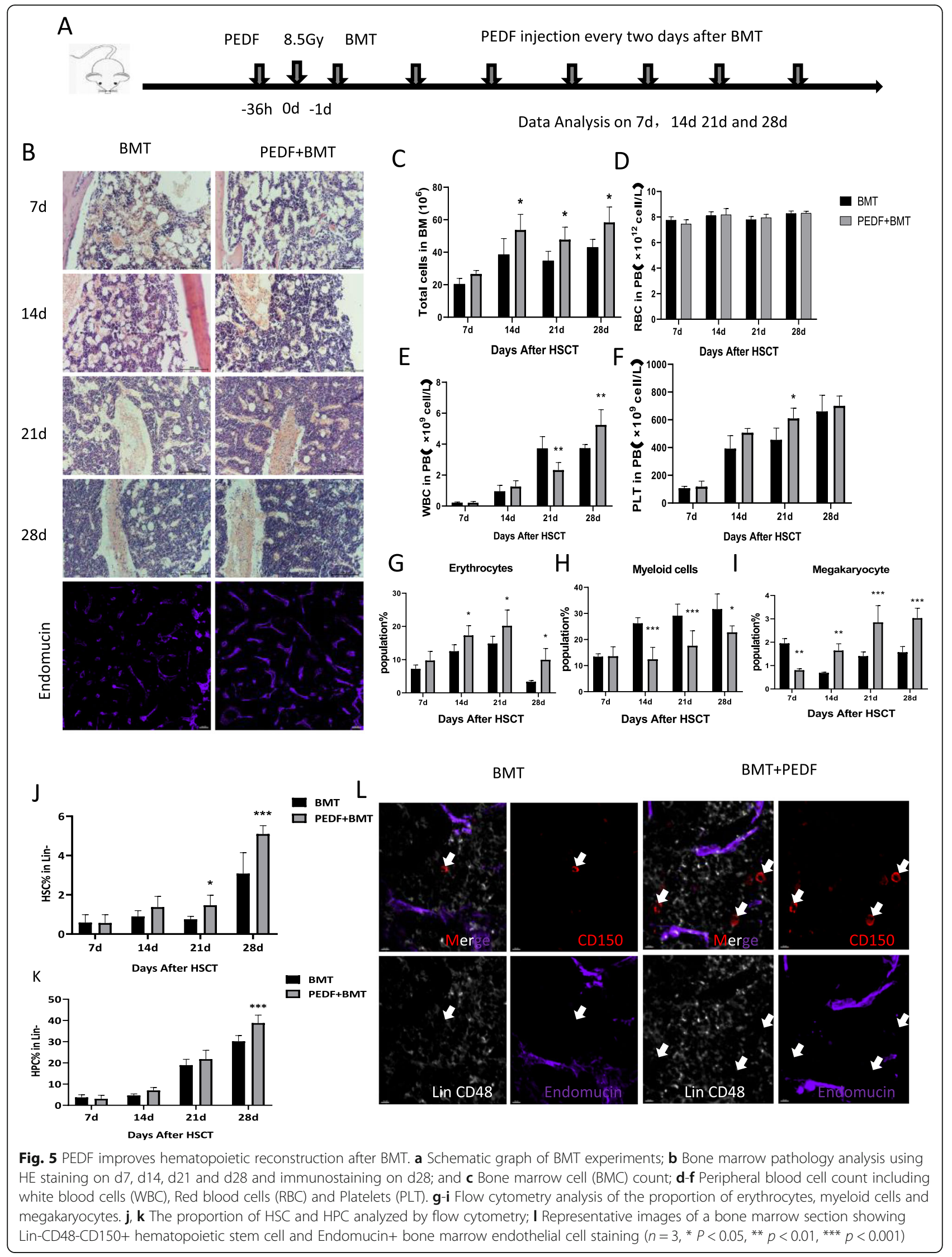




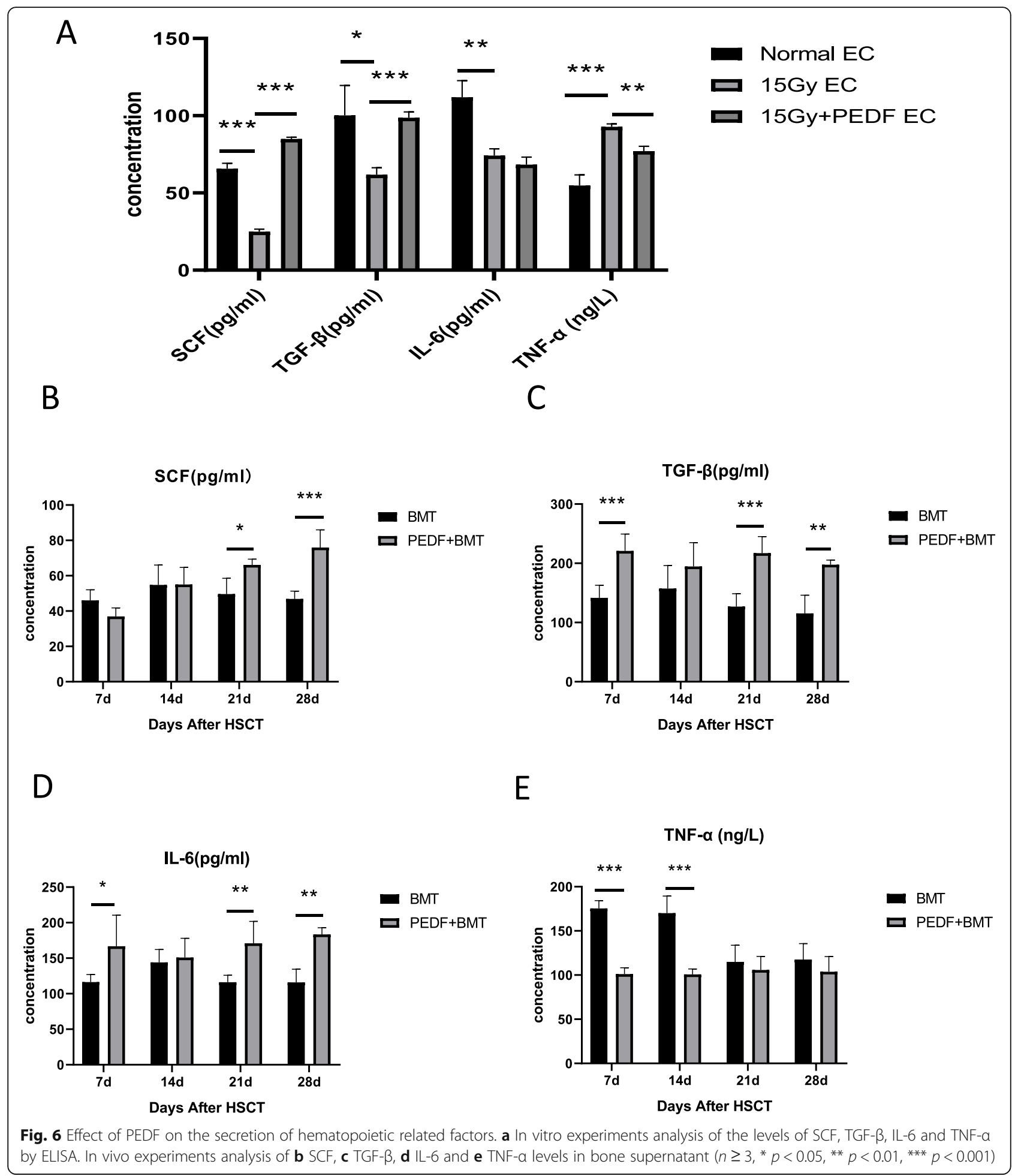

have shown that PEDF as a regulator of stem cells can promote the self-renewal of neural hepatocytes [35, 36], but its direct effect on hematopoietic stem cells is minimal, and we have found that PEDF can reduce bone marrow pathological damage and bone marrow microvascular damage. It is suggested that PEDF may repair bone marrow microvessels and then promote hematopoietic reconstruction.

This study also found that the permeability of the injured endothelial cells changed significantly and the PEDF recombinant protein is a secreted protein that is closely related to endothelial permeability. We then 
assessed the effect of PEDF on injured endothelial function in vitro and found that PEDF can inhibit the permeability changes of injured endothelial cells, so as to maintain endothelial function. Endothelial cell permeability is mainly composed of the paracellular pathway (cell junction) and the transcellular pathway, of which the paracellular pathway is the main pathway [37]. The paracellular pathway mainly has two forms of tight junctions (TJs) and adhesive junctions (AJs). TJ is mainly composed of occludin and claudin. These two types of transmembrane proteins are connected to the actin cytoskeleton through a locking protein (ZO-1) [38]. The increase in vascular permeability caused by $\mathrm{TJ}$ is mainly caused by degradation or phosphorylation of related proteins, in which occludin is hydrolyzed to inactive fragments and silk / threonine phosphorylation of ZO-1 protein is closely related to increased permeability [38]. AJs are considered to be the main connection of the peripheral microvascular system. It is mainly composed of the transmembrane protein vascular endothelial cadherin (VE-cadherin), which is fixed on the actin cytoskeleton by catenin $(\alpha-, \beta-, \gamma$-catenin or bridging protein p120) [38]. The disassociation of AJs can lead to increased cell permeability. This study found that the levels of ZO-1, VE-cadherin and occludin in the injured endothelial cells were decreased after irradiation, suggesting that irradiation can increase the permeability of vascular endothelial cells, consistent with previous studies $[39,40]$. Mouse bone marrow microvascular endothelium serves as a portal for hematopoietic cells in the bone marrow. The increased microvascular endothelial permeability caused by irradiation may be related to failure implantation of hematopoietic stem cells and bone marrow hemorrhage after irradiation injury in mice. However, PEDF can inhibit the increase of endothelial permeability [21] and promote the colonization of implanted hematopoietic stem cells.

Studies have suggested that PEDF has multiple receptors (PEDF receptor, PEDFR) and it binds to different receptors to play different functions [41-46]. For example, PEDF interacts with the phospholipase A2 receptor, which can exert anti-oxidation and anti-apoptosis or cytoprotection effects; PEDF interacts with laminin receptor (LR) to exert anti-angiogenesis effect and inhibit endothelial cell migration. In addition, the interaction between PEDF and ATGL is related to insulin resistance. However, this study found that the interaction of PEDF with the receptor ATGL might promote the expression of VE-cadherin and occludin, which are related to the permeability of endothelial cells. In addition, this study also found that hematopoietic related factors were changed significantly after addition of PEDF, suggesting that hematopoietic related factors might participate in the effect of PEDF on repairing damaged endothelial cells and promoting hematopoietic reconstruction.

\section{Conclusions}

In conclusion, our study demonstrates that PEDF inhibits the increased endothelial permeability induced by irradiation and reverse the inhibitory effect of injured endothelial cells on hematopoietic stem cells and promote hematopoietic reconstruction.

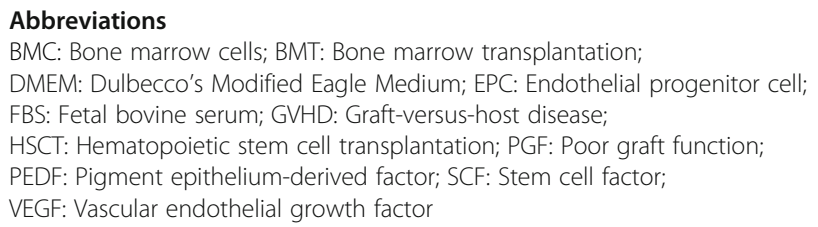

Acknowledgements

We thank Dr. Meng Zhao (Sun Yat-Sen University, Guangzhou, China) for technical support.

\section{Conflict of interest}

The authors have declared that no conflict of interest exists.

\section{Authors' contributions}

W. Ju, W. Lu, L. Ding, Y. Bao, F. Hong, Y. Chen, H. Gao, X. Xu, G. Wang, W. Wang, X. Zhang, C. Fu, K. Qi, and Z. Li performed the experiments and analyzed data. K. Xu, J. Qiao, and L. Zeng designed the study, analyzed and interpreted data and wrote the manuscript. The author(s) read and approved the final manuscript.

\section{Funding}

This study was supported by National Natural Science Foundation of China [Grant number 31872795, 81700178 and 81770223]; Jiangsu Province's Key Provincial Talents Program under Grant [number ZDRCA2016054]; Natural Science Foundation of Jiangsu Province under Grant [number BK20170259]; Major Basic Research Project of the Natural Science Foundation of the Jiangsu Higher Education Institutions under Grant [number 17KJA320008]; China Postdoctoral Science Foundation Grant [number 2018 M632380]; Jiangsu Postdoctoral Science Foundation under Grant [number 1701064B]; Postgraduate Research \& Practice Innovation Program of Jiangsu Province under Grant [number KYCX19_ 2247].

\section{Availability of data and materials}

All data generated or analysed during this study are included in this published article.

\section{Ethics approval and consent to participate}

This study was approved by the animal ethnic committee of Xuzhou Medical University.

\section{Consent for publication}

Not applicable.

\section{Competing interests}

Authors have no conflict of interests to declare.

\section{Author details}

${ }^{1}$ Blood Diseases Institute, Xuzhou Medical University, Xuzhou, China. ${ }^{2}$ Key Laboratory of Bone Marrow Stem Cell, Jiangsu Province, Xuzhou, China. ${ }^{3}$ Department of Hematology, the Affiliated Hospital of Xuzhou Medical University, Xuzhou, China. ${ }^{4}$ Medical Center of Hematology, Xinqiao Hospital, Third Military Medical University, Chongqing, China.

Received: 19 February 2020 Accepted: 26 August 2020

Published online: 01 September 2020

References

1. El Fakih R, Ahmed S, Alfraih F, Hanbali A. Hematopoietic cell transplantation for acute lymphoblastic leukemia in adult patients. Hematol Oncol Stem Cell Ther. 2017;10(4):252-8. 
2. Jethava YS, van Rhee F. Transplantation for multiple myeloma. Cancer Treat Res. 2016;169:227-50.

3. Takami A. Hematopoietic stem cell transplantation for acute myeloid leukemia. Int J Hematol. 2018;107(5):513-8.

4. Kong Y. Poor graft function after allogeneic hematopoietic stem cell transplantation-an old complication with new insights (). Semin Hematol. 2019;56(3):215-20.

5. Masouridi-Levrat S, Simonetta F, Chalandon Y. Immunological basis of bone marrow failure after allogeneic hematopoietic stem cell transplantation. Front Immunol. 2016;7:362.

6. Kong $Y$, et al. The bone marrow microenvironment is similarly impaired in allogeneic hematopoietic stem cell transplantation patients with early and late poor graft function. Bone Marrow Transplant. 2016;51(2):249-55.

7. Pinho $\mathrm{S}$, et al. Lineage-biased hematopoietic stem cells are regulated by distinct niches. Dev Cell. 2018;44(5):634-641 e634.

8. Wang $\mathrm{A}$, Zhong $\mathrm{H}$. Roles of the bone marrow niche in hematopoiesis, leukemogenesis, and chemotherapy resistance in acute myeloid leukemia. Hematology. 2018;23(10):729-39.

9. Bowers E, et al. Granulocyte-derived TNFalpha promotes vascular and hematopoietic regeneration in the bone marrow. Nat Med. 2018;24(1):95102.

10. Gori $\mathrm{L}$, et al. Endothelial cells promote expansion of long-term engrafting marrow hematopoietic stem and progenitor cells in primates. Stem Cells Transl Med. 2017;6(3):864-76.

11. Guo P, et al. Endothelial jagged-2 sustains hematopoietic stem and progenitor reconstitution after myelosuppression. J Clin Invest. 2017;127(12): 4242-56.

12. Qiao J, et al. Endothelial progenitor cells improve the quality of transplanted hematopoietic stem cells and maintain longer term effects in mice. Ann Hematol. 2017;96(1):107-14.

13. Salter $A B$, et al. Endothelial progenitor cell infusion induces hematopoietic stem cell reconstitution in vivo. Blood. 2009;113(9):2104-7.

14. Hassanshahi M, Hassanshahi A, Khabbazi S, Su YW, Xian CJ. Bone marrow sinusoidal endothelium: damage and potential regeneration following cancer radiotherapy or chemotherapy. Angiogenesis. 2017;20(4):427-42.

15. Passaro D, et al. Increased vascular permeability in the bone marrow microenvironment contributes to disease progression and drug response in acute myeloid leukemia. Cancer Cell. 2017;32(3):324-341 e326.

16. Qiao J, et al. Evaluation of the effects of preconditioning regimens on hepatic veno-occlusive disease in mice after hematopoietic stem cell transplantation. Exp Mol Pathol. 2015;98(1):73-8.

17. Zeng $L$, et al. Vascular endothelium changes after conditioning in hematopoietic stem cell transplantation: role of cyclophosphamide and busulfan. Transplant Proc. 2010;42(7):2720-4.

18. Zeng L, Yan Z, Ding S, Xu K, Wang L. Endothelial injury, an intriguing effect of methotrexate and cyclophosphamide during hematopoietic stem cell transplantation in mice. Transplant Proc. 2008;40(8):2670-3.

19. Zeng $L$, et al. Irradiation is an early determinant of endothelial injury during hematopoietic stem cell transplantation. Transplant Proc. 2008;40(8):2661-4.

20. Park K, Jin J, Hu Y, Zhou K, Ma JX. Overexpression of pigment epitheliumderived factor inhibits retinal inflammation and neovascularization. Am J Pathol. 2011;178(2):688-98.

21. Zhang $\mathrm{H}$, et al. PEDF improves cardiac function in rats with acute myocardial infarction via inhibiting vascular permeability and cardiomyocyte apoptosis. Int J Mol Sci. 2015;16(3):5618-34.

22. Zhang $\mathrm{H}$, et al. PEDF and 34-mer inhibit angiogenesis in the heart by inducing tip cells apoptosis via up-regulating PPAR-gamma to increase surface FasL. Apoptosis. 2016;21(1):60-8.

23. Elahy M, Baindur-Hudson S, Cruzat VF, Newsholme P, Dass CR. Mechanisms of PEDF-mediated protection against reactive oxygen species damage in diabetic retinopathy and neuropathy. J Endocrinol. 2014;222(3):R129-39.

24. Kadoya M, Tamoto E, Shichinohe T, Hirano S. Pigment epithelium-derived factor inhibits the growth of human esophageal squamous cell carcinoma by suppressing neovascularization. Hokkaido Igaky Zasshi. 2015;90(1):17-29.

25. Seki $R$, et al. Pigment epithelium-derived factor (PEDF) inhibits survival and proliferation of VEGF-exposed multiple myeloma cells through its antioxidative properties. Biochem Biophys Res Commun. 2013;431(4):693-7.

26. Fitchev $P$, Chung C, Plunkett BA, Brendler CB, Crawford SE. PEDF \& stem cells: niche vs. nurture. Curr Drug Deliv. 2014;11(5):552-60.
27. Sheikpranbabu S, Haribalaganesh R, Lee KJ, Gurunathan S. Pigment epithelium-derived factor inhibits advanced glycation end products-induced retinal vascular permeability. Biochimie. 2010;92(8):1040-51.

28. Tong JP, Yao YF. Contribution of VEGF and PEDF to choroidal angiogenesis: a need for balanced expressions. Clin Biochem. 2006;39(3):267-76.

29. Cai J, et al. PEDF regulates vascular permeability by a gamma-secretasemediated pathway. PLoS One. 2011;6(6):e21164.

30. Ma S, et al. Pigment epithelium-derived factor alleviates endothelial injury by inhibiting Wnt/beta-catenin pathway. Lipids Health Dis. 2017;16(1):31.

31. Hooper AT, et al. Engraftment and reconstitution of hematopoiesis is dependent on VEGFR2-mediated regeneration of sinusoidal endothelial cells. Cell Stem Cell. 2009;4(3):263-74.

32. Ramalingam P, Poulos MG, Butler JM. Regulation of the hematopoietic stem cell lifecycle by the endothelial niche. Curr Opin Hematol. 2017;24(4):28999.

33. Li XM, Hu Z, Jorgenson ML, Wingard JR, Slayton WB. Bone marrow sinusoidal endothelial cells undergo nonapoptotic cell death and are replaced by proliferating sinusoidal cells in situ to maintain the vascular niche following lethal irradiation. Exp Hematol. 2008;36(9):1143-56.

34. Rorby $E$, et al. The stem cell regulator PEDF is dispensable for maintenance and function of hematopoietic stem cells. Sci Rep. 2017;7(1):10134.

35. Chojnacki A, Weiss S. Pigment epithelium-derived growth factor: modulating adult neural stem cell self-renewal. Nat Neurosci. 2009;12(12): 1481-3.

36. Ramirez-Castillejo C, et al. Pigment epithelium-derived factor is a niche signal for neural stem cell renewal. Nat Neurosci. 2006;9(3):331-9.

37. Komarova Y, Malik AB. Regulation of endothelial permeability via paracellular and transcellular transport pathways. Annu Rev Physiol. 2010;72: 463-93.

38. Komarova YA, Kruse K, Mehta D, Malik AB. Protein interactions at endothelial junctions and signaling mechanisms regulating endothelial permeability. Circ Res. 2017;120(1):179-206.

39. Gabrys D, et al. Radiation effects on the cytoskeleton of endothelial cells and endothelial monolayer permeability. Int J Radiat Oncol Biol Phys. 2007; 69(5):1553-62

40. Wang $\mathrm{H}$, et al. Gamma radiation-induced disruption of cellular junctions in HUVECs is mediated through affecting MAPK/NF-kappaB inflammatory pathways. Oxid Med Cell Longev. 2019;2019:1486232.

41. Yao S, et al. Pigment Epithelium-Derived Factor (PEDF) protects osteoblastic cell line from glucocorticoid-induced apoptosis via PEDF-R. Int J Mol Sci. 2016;17(5):730.

42. Zhao Q, et al. PEDF improves cardiac function in rats subjected to myocardial ischemia/reperfusion injury by inhibiting ROS generation via PEDFR. Int J Mol Med. 2018:41(6):3243-52.

43. Zhou Z, et al. PEDF inhibits the activation of NLRP3 inflammasome in hypoxia cardiomyocytes through PEDF receptor/phospholipase A2. Int J Mol Sci. 2016;17(12):2064.

44. Zhuang W, et al. PEDF and PEDF-derived peptide 44mer inhibit oxygenglucose deprivation-induced oxidative stress through upregulating PPARgamma via PEDF-R in H9c2 cells. Biochem Biophys Res Commun. 2016; 472(3):482-8.

45. Wang $X$, et al. PEDF attenuates hypoxia-induced apoptosis and necrosis in H9c2 cells by inhibiting p53 mitochondrial translocation via PEDF-R. Biochem Biophys Res Commun. 2015;465(3):394-401.

46. Zhang $\mathrm{H}_{\text {, et }}$ al. PEDF and PEDF-derived peptide 44mer stimulate cardiac triglyceride degradation via ATGL. J Transl Med. 2015;13:68.

\section{Publisher's Note}

Springer Nature remains neutral with regard to jurisdictional claims in published maps and institutional affiliations. 